\title{
Research on the regional sustainable theory of modern architecture in traditional thinking
}

\author{
Bo Zhang ${ }^{1}$
}

Environmental Design Department,School of Architectural and Civil Engineering,Shenyang City University, No.2,Wutong Street,Sujiatun District, Shenyang City, Liaoning Province, China

\section{Corresponding author: Bo Zhang}

\begin{abstract}
Key words: regional feature; traditional culture; regional architecture ;sustainable theory
\end{abstract}
\begin{abstract}
Architecture should be the synthesis of time and space. It shows the features of technologies, environments and cultures, however, it should have special character of the regional and cultural means. Traditional architecture based on impression, and conveyed the culture and awareness of China. So, regional architectures not only embody symbol skins of architecture, but also affect the impression and development in sustainable architecture. Therefore, the architectural understanding of traditional Chinese cultural thinking should also continue to affect the expression and development of modern regional architecture in the sustainable sense.
\end{abstract}

\section{传统思维下的现代建筑地域化可持续理论研究}

\author{
张博 1 \\ 1 沈阳城市学院环境设计系, 沈阳, 辽宁, 中国 \\ 810154092@qq.com \\ 通讯作者:张博
}

关键词：地域性；传统文化；地域建筑；可持续理论

中文摘要. 建筑应该是特定的时空耦合。建筑所呈现出的技术、环境及文化等特征，在建筑 层面也应具有特定的地域特点与文化含义。传统的中国建筑在赋予情感的基础上，也是传统 文化与意识的集中反映。那么，地域建筑所应表征出的便不应仅是符号作用下的表皮; 对于 地域可持续化的理解也不应仅是脱离地域环境的存在。因此, 对于中国传统文化思维下的建 筑理解，也应持续地影响着现代地域性建筑在可持续意义层面的表达与发展。

\section{1. 地域建筑的生命特质}

建筑自古以来作为人类生存与发展的掩体居所，是与自然长期相互作用的产物。作为建 筑而言, 其最主要特点, 便是受到自然、社会、文化等多重因素影响下, 所体现出的地域文 化特性。而这种地域性所呈现出的是人们对生存的强烈愿望。因此, 出于安全、生存等方面 的考虑, 并掺杂对自然的初始理解, 巢居抑或穴居的形式成为了人类初始的空间选择。这些 从对图腾的崇拜, 到自然的认识与理解所孕育出的传统文化哲学, 都无疑展现了在自然影响 下所呈现出的特定的建筑形式与结果, 对于客观自然的考虑以及建筑与自然相结合的方式, 因此也成为建筑地域性产生和发展的重要缘由。在早期蒙昧的原始社会, 基于人们对自然的 崇拜态度。通过祭祀以求获得人的生存权利, 成为起初生命意识的体现; 自然雨露所衍育的 
农作物, 产生了人对于“生”的思考, 同时也将自然赋予了生命意识, 促使人们从多方面去探 寻人与自然之间的神秘关系。“生生之谓易也”、“天人合一”等朴素哲学观念, 抑或是出于原 始自然观所形成的传统风水术、堪舆术等也都体现出传统自然观下的人在建筑中所体现的对 生命的态度、对自然法则的尊重, 即“人法地, 地法天, 天法道, 道法自然” (《道德经·道经》 第二十五篇）。人需要取法于天，以此说明人与自然法则下“道”的关系。而基于风水文化的 作用下, 背山面水、形势说等理论的提出, 又对早期的传统建筑营造及方向给予指导作用并 对聚落乃至城市规划层面有着深刻的意义。如古代春秋时期所建圔闾大城（今江苏苏州市） 的营建过程中，吴国大臣伍子胥提出“相土尝水，象天法地”, 即通过取法于自然, 根据客观 的自然情况进行城市的选址与规划建设。其中重点对水域的形成以及脉络发展情况、当地土 质的肥沃程度等方面进行逐一的考察和分析; 对城池还设置了与天象之数相符的城门......这 都成为了以风水观念导则下的城池建设的完美体现, 使之具有与当地文化、自然相对应的地 域含义。最终, 受人类长期形成的生活行为及习俗影响, 从文化、社会、自然环境等多个层 面共同施加作用，焕发出建筑的地域特质与独特的生命活力。

\section{2. 地域性建筑的传统复归}

受传统生活方式及社会习俗所形成的文化适应性影响, 人们对空间认知、营造的选择具 有强烈的本土地域特征, 这也是由于地域不平衡性, 所体现出的必然的文化多样性与人文情 感体现。在历史发展过程中所衍生出的地域建筑, 经文化的进化与发展演变, 是一种基于地 域性的生态行为, 是世代传承文化的物化结果, 这具有强烈的文化完整性与延续性, 正如所 处纬度不同的影响, 导致环境的差异, 使我国在以黄河为界, 其南北方在具体的空间处理上 呈现出对阳光热量汲取上有着截然不同的态度：黄河以北的建筑以围合成的开敞院落为主, 以求最大限度的利用阳光所带来的能量; 黄河以南的建筑却由于温热气候的作用下, 大多以 制造阴影、实现由热压差而导致的空气上下流通的天井为主要的能量控制手段, 从而以一种 建筑惯性的形式予以传承。可见, 这种在自然作用下的建筑, 在体现出文化地域的独特个性 的同时, 也展现出其文化的延续性。这是自然环境作用下必然发生的结果, 从而在与自然相 互作用的过程中体现着建筑的生态理念与人文情感。而随着科技发展, 也带来更大程度上的 技术自由: 交通发展带来的能量转移, 摆脱了对当地资源的依赖; 通过进行空间舒适度的技 术控制, 使空间拥有了人们所必须的生活条件.....这种基于 20 世纪初的建筑观, 却往往带有 将建筑与地域关系相分离的倾向, 在对于技术崇拜的过程中, 也将技术置于脱离自然的独立 地位。这将势必导致对建筑行为的反作用力, 形成内心情感与自然的远离, 造文化的混乱和 不可持续。

从早期对“天人合一”与上下与天地同游”的境界追求，转而将客观世界置于分离状态下 对自然的强制协调性。可见, 这种转换并非仅是语义的改变, 而是对建筑的观念与认知的误 区。深层生态学理论认为“环境危害应从改变现存的价值观念及生产消费模式, 用“绿色”文明 取代工业文明的“灰色”文明，用“节俭社会”代替“富裕社会”’[1]，显然，这是对于当前对技术的 客观认知状态下的纠错行为, 这与建立在地域环境传统思维下的建筑营造意识存在着一定的 共通性。因此, 这种传统意识中的“自然之道”, 其强调对于自然的和谐一致基础上的和谐与 共生, 是一种自然的可持续以及对空间生态性的把握对于现代建筑的地域性回归而言, 不仅 是一种地域和文脉的回归, 也是可以成为对于未来建筑发展的启示。可见，“师法自然”不仅 是从宏观上的遵循自然, 也是延续地域文化的生态建筑, 重回人与自然的接触得一种可行的 方式与手段。

1 赵红州.生态文化应急行. 人类与森林, 1997,4. 


\section{3. 现代地域建筑的可持续设计架构}

建筑的地域性需要在普遍性与地方性之间把握动态的平衡, 这是基于长期的环境及其所 建立起的文化层面上的自觉选择。实现建筑生态的动态平衡与实现可持续效益, 需要从经济、 文化、社会等多个维度进行分析与综合的研究。对于建立特有地域环境的特质, 把握城市的 文脉及地域特征, 更需要注重从整体层面形成新的环境需求。

\section{1 注重地域特征的延续}

建筑是具体环境中对地域资源与特性的理性、感性二者共同作用的结果。人们经过长期 的探索逐渐掌握并学会运用自然环境资源, 并使技术成为地域文化存在, 它掺杂着地域间特 有的交流方式与情感, 赋予了建筑特殊的人文含义。这是地域特征与传统生活方式的延续, 并体现着对于人性的肯定。工业革命后的技术飞跃，传统观念并不能对现代人对于物质等方 面不断的追求给予及时的反馈与纠正, 加之资源的匮乏, 得技术在缓解这种激化的矛盾的同 时, 也相对应造成了“地域建筑创作中价值理性与工具理性的背离, 出现了以激进意识形态上 的创新为目的的技术非理性现象”。[2]

经长期探索的地域建筑，其本质上是对当人社会性的集中体现，并在此基础上赋予多元 的含义。因此, 基于传统自然条件下的地域性生态发展前提下, 从人的社会性层面恢复地域 的多样性、传承地域文化也是建筑在设计过程中不可回避的问题。对于当下我国建筑而言, 技术所带来的在建筑上的变革，不应成为日后我国建筑发展的达摩克利斯之剑。对技术所赋 予权利的盲目崇拜, 将会导致生态的混乱, 以至于威胁人类生存。因此, 建筑的发展, 更应 体现出一种对于尊重地域环境的自觉意识。正如哈桑·法赛在新古纳尔村的改进型土拱住宅实 践中, 经对传统材料以及构造方式的科学评价后, 理性的“用灰泥夹杂稻草制成轻型砖, 以扁 斧砌拱顶和穹顶”[3]，这不仅是建筑师以最为质朴的手段强调建筑在地域环境中的地位，也在 创造性的强调“文化的真实性”, 即“只有根植于当地地理、文化环境中的本土建筑才是社会建 筑的真实表达”[4]。可见, 在对于传统的文化情感的传承过程中, 防止文化系统的生态失调, 建筑师更应以开放的姿态，在理解借鉴传统与自然环境相作用的经验与技术的同时，将地域 建筑理解为功能、经济、文化等因素共同作用的结果, 来诠释地域文化所表达的社会层面意 义。这是以理性的方式把握人与环境的关系, 使建筑富有深刻内涵的必然所在。因此, 建筑 师应理解建筑风格下与地域的关系, 把握地域环境最真实的现实, 表达地域建筑所传达的文 化深刻性。

\section{2 传承可持续的共生思想}

生态学作为“研究有机体与其周围环境——包括非生物环境和生物环境相互关系的科 学”,[5], 势必在人其作用的环境系统中发挥着重要的意义。从我国早期文化遗址, 可见在边际 效应所维持的物种多样性状态下，人类始终与其所在的自然环境相互发生作用并保持自身的 生存与发展。在人类占据生态空间的生态位时, 实质也进入了自然的循环系统的过程, 在竞 争中求得共存与发展。在对于自然极度依赖的前提下，对自然的态度便是基于对自然环境的 自觉反应。从而衍生出中国的朴素文化哲学——基于世界的本体“道”的概念。“道”是高度概 括的自然, 自然的规律, 万物的生长都遵循于自然之“道”。因此, “道”意味着在道德伦理层 面基础上对于万物的尊重。“竭泽而渔”（《吕氏春秋·义赏》）至“置于爱民, 以下至鸟兽昆 虫莫不爱”。( 《春秋繁露. 仁义法》) 都体现出在中国传统体验与自然和谐的基础上, 这种 求“天地之和”所追求的正是一种万物共生的尊重。作为中国传统文化内涵的一部分, 现代地 域建筑在创作过程中, 却往往忽视了对于自然的理解, 导致地域建筑的片面符号化与形式主

2 章明，张姿.当代中国建筑的文化价值认同分析.时代建筑，2009（3）：18-23

3 刘先觉等.生态建筑学. 北京: 中国建筑工业出版社 2009.05: 62

4 林南.在神秘的面纱背后一一埃及建筑师哈桑・法赛评析.世界建筑, 1992,6:67-72

5 刘先觉等.生态建筑学. 北京: 中国建筑工业出版社 2009.05: 40 
义泛滥。这本质上是对技术层面的认知的自觉模糊所导致, 片面强调技术的权威而忽视对于 环境与建筑关系的本源关系, 即建筑是对于环境的有效反映。可见, 生态问题与环境问题的 解决更需要建立在建筑与环境的科学与理性基础上的正确认知。只有通过人与自然的相互作 用基础上, 才能真正的焕发地域建筑的生命活力, 建立环境的和谐整体观与共生意识, 提高 建筑在生态系统中的综合效益。如新加坡杨经文先生, 便是基于生态观念将共生思想在其雪 兰莪州 IBM 大厦项目中予以诠释。由于建筑处于炎热的气候带, 因此建筑设置大量的开口, 将植物进行建筑的表皮覆盖, 进行建筑的生态系统调节。同时, 绿化也充当了城市生态绿网 的重要节点, 减少对于生物生存的障碍, 也是对生物友善的建筑意识体现。

“共生”是将建筑视为自然有机体的一部分，这需要将其参与环境的生态系统循环之中来 实现。这不仅是地域建筑的要求所在, 也是建筑师秉持对生态环境意识的原则操守。在文化、 经济、社会等多个因素共同作用于建筑系统的设计过程中, 提高自然的自循环系统的能力与 生态平衡, 从建筑全生命周期的角度考虑与生态系统的共生关系, 同时, 这也是传统观念在 现代地域建筑设计中的体现。可见, 在基于对自然万物的理解基础上, 结合气候、文化、环 境、材料的地域建筑, 更应强调一种对于自然生态系统的认知自觉, 也是一种对于传统自然 意识的深刻理解与传承。

\section{3 强调生态系统的动态性特征}

绿色建筑理论将建筑视为有机的循环系统。与自然生态系统相类似的, 建筑也应具有生 态的自循环特征。因为建筑是处于多种影响因子交织作用的结果, 而作用于建筑的不同影响 因子都是处于特定的“时一一空” 状态下的特殊产物。因此, 现代绿色建筑既具有稳定因子作 用下的稳定性, 同时也体现出某种程度的动态性, 即允许当下的现实使用要求, 也可以在一 定状态下, 进行能量的输入与输出, 保证未来的动态发展需要。实现人、建筑与自然环境的 三者统一，形成整体范围内的稳定状态。

在当代地域建筑的发展过程中，实质也是基于传统文脉及地域特性的延续。这种结合自 然的传统建筑观念, 是在把握环境的整体性前提下, 进行的传统建筑空间营造。如中国传统 村落, 在对选定区域进行风水的相地及选址过程后, 建筑便会以村落为中心, 进行村落的规 模扩张, 从而形成由点及面的增长网络。而这种增长会始终把握对于建筑与自然的和谐, 形 成具有一定程动态开放特征的生态梯度。可见, 传统建筑在其前期以及营造的过程中, 对于 自然一建筑一人三者关系需要留有阈值, 来保证其应对不同的条件。再如传统的干阑式 建筑, 便体现了对地域环境的适应性的结果: 架起的房屋可提供居住功能; 下部露空之处可 做储藏与蓄养之用, 产生的通风以保持凉爽, 与热工性能良好的木材共同作用下, 保证了室 内良好的热适应性。而在大片水域之处, 干阑建筑也通过其极强的适应性来体现其开放性特 点。“竹楼的竹鏼多空隙, 且系绑于梁上, 洪水泛滥时, 可将其取下减少浮力, 俟洪水退后再 铺上" "[6]。可见, 在现代地域建筑的创作过程中, 更是需要汲取传统环境观及建筑观的态度, 加强对环境认知的理性把握, 使得空间具有一定的动态开放性的特征, 并理性把握对现有空 间的认知，从而使得建筑于环境具有更为密切的联系。同时，这种认知，还应考虑新科技、 空间延展性等因素将传统与现代因素在现有条件下形成互为补充, 提供给科技等因素提供空 间, 才可真正的实现地域建筑的可持续性。可见, 建筑在具有一定宽容度的空间下, 才能形 成建筑与环境的统一, 将现在与未来相对话。因此, 呈现在地域建筑上的所体现出的可持续 性, 这不仅需要在建筑本身中寻找答案, 更需要在对整体环境充分认识的基础上, 提供给未 来变化发展一定空间, 才可形成日后建筑活力与发展的物质基础。可见, 动态性特征也是现 代地域建筑在未来可持续发展的题中之意。

6 朱馥艺.干阑一一中国西南传统民居初探[D].华中理工大学, 1997:15, 转引自云南省设计院《云南民居》

编写组.云南民居[M].北京: 中国建筑工业出版社, 1986 


\section{4 突出大众的参与意识}

由于大众的参与，使得建筑向“真”的方向发展。人类是以特殊的方式介入到原生的生态 系统之中, 这在传统建筑实例中可以找到多个答案。早期人类根据对环境的模糊理解, 在逐 步试错的过程中形成了具有地域特质的建筑语言与形式。但是, 这种地域建筑所呈现出的并 非仅是地域的形式面貌，同时也体现着当地公众的共识与价值观念。

在传统的文化传承过程中, 在建筑形成的空间里体会通过注入情感, 形成地域性甚至是 民族特征, 也会随着技术与自然的相互的作用下，传统建筑以法则及文化适应的方式保持着 其建筑的惯性。可见, 人在环境中的有意义参与, 才使建筑赋予了真正的内涵。因此, 对于 建筑师而言, 一个好的建筑作品的实现, 往往是将建筑的设计行为建立在对人活动的高度理 解基础上, 即建立在对大众的积极参与和认知的基础上, 保持着对于地域性的理解与环境体 验关系, 最终将环境、经济、文化等多个因素以空间的方式合理的呈现。同时, 生态学理论 对建筑全生命周期的考虑是对建筑是否可持续的科学评价也给予建筑师对地域建筑的设计提 供了新的启示。对使用者而言, 室内的使用阶段占据了其生活的绝大部分时间。因此, 人作 为建筑的重要影响因子, 通过对于建筑的时刻参与, 来完成对建筑的影响。可见, 行为会以 自己对环境的现实需要, 对环境施加作用。因此, 为了实现行为主体的意义与需求, 更需要 建筑师对行为主体, 即空间的使用者的需求进行深入的考察与了解, 以保证建筑对于人产生 意义, 从而使建筑实现人与环境的联系。这包括受所处地域环境影响下的心理状态、生活方 式、文化及习俗等多种因素, 可见公众对于地域建筑的可持续设计思路的把握与方向的引导 也起着关键性作用。在特定的地域环境中理解真正的文脉及历史性, 避免了建筑师的主观意 识下对于地域建筑设计的主观臆断倾向。因此, 在全球化与地域性, 现代性与传统性交融的 今天, 建筑师更应转换设计思路, 提高建筑师的责任意识, 这并非仅是对于建筑浅层的研究, 更应通过于公众的对话, 深挖地域环境的特质, 使建筑师与公众形成优势互补, 扩大建筑师 的设计思路与视野。实现从建筑师狭险的主观设计意识转变为真正为人而设计, 为地域而设 计, 这也是以人为本的要求所在。

公众参与是保持建筑生命力的重要部分, 这将使建筑师更大的发挥设计的主体意识以及 价值判断能力。通过大众的设计全过程参与, 实现设计师与公众的平等对话, 才不会使得建 筑成为空中楼阁。从而从环境整体性上审视地域建筑的可持续性表现。从而实现具有地域个 性的多样化、多层次的地域建筑，这也是地域建筑可持续设计的精髓。

\section{4. 结语}

基于环境所要求, 地域性的建筑设计才是建筑的本质体现。随着技术变革的不断深化, 提供了方便的同时, 却疏远了最真的现实。现代建筑观只是对于当今资源危机、提高生活条 件的紧急避难所。但是, 生存并不意味着健康, 对于资源的思考还涉及到技术伦理及可持续 问题。千百年来, 反复的试错所孕育建筑的地域性, 其作为文化现象, 所需要解决的并非仅 是遮蔽功能, 还是一种文化多样性的反映与为了更好生存的生态空间的处理手段。因此, 在 现代地域建筑的可持续研究的同时, 传统文化承载下的建筑, 也不失为一种对自然可持续之 道的新视角。

\section{References}

[1] Xianjue Liu,et at.Architecture of Ecology,Beijing : China Architecture \& Building Press, pp. 40-62,2009

[2] Hongzhou Zhao.Ecological culture should take urgent action,Forest \& Humankind,vol.4, pp. 4-10, 1997

[3] Zhang Ming, Zhang Zi. Analysis on the Culture Value Identity of Contemporary Architecture in 
China.Time Architecture, vol.5, pp. 18-23, 2009

[4] Lin Nan.Behind the enigmatic veil -- an analysis by Egyptian architect HassanFathy, World Architecture, vol.6, pp. 67-72, 1992

[5] Xinyi Zhu,.Ganlan_-A preliminary study on traditional vernacular architecture in southwest China, Wuhan:Huazhong University of Science and Technology press, vol.15,1997

[6] Mei Hongyuan, Zhang Xiangning, Zhu Ying.Back to contemporary Chinese regional architecture creation.Architectural Journal, vol.9, pp. 106-109, 2010.

[7] Mouchang .The ecological and ethical value of ancient Chinese philosophy.History of Chinese Philosophy, vol Z1, pp. 33-42, 1996

[8] LI Tian-dao."HE" :The Theory of Existenzialen Constitution in Traditional Chinese Eco-Aesthetics.Journal of Guizhou Normal Uni versit y (Social Science), vol 01, pp. 100-103, 2004

[9] WANG Qian."Control Technique by Tao"___Ethics of Technology in Pre-Qin and its Modern Significance (p.8),Journal of Dialectics of Nature, .vol 30:173, pp. 8-14, 2008

[10] Hengchun Xu.Ecological Aesthetics, Xi'an:Shanxi People’s Education Press, 2000

[11] Qiheng Wang,et at..Research to Geomantic Omen theory .Tianjin : Tianjin University Press, 2005

[12] Sha Run.Outlook on The Nature and Origins of The Culture of Traditional Residential Architecture in China,Human Geography,vol 12:3,pp. 25-29,1997

[13] Haoming Zhou, Theory of Sustainable Interior Environment Design.Beijing :China Architecture \& Building Press, 2011 